\title{
Development of Oil Damper Test Equipment Capable of Simulating the Actual Conditions of Railway Vehicles
}

\author{
Nobuyuki WATANABE \\ Assistant Senior Researcher, \\ Running Gear Laboratory,
}

\section{Mineyuki ASAHINA}

Researcher,

Vehicle Noise \& Vibration Laboratory, Vehicle Noise \& Vibration Laboratory,
Tadanobu IIDA

Former Researcher, Vehicle Dynamics Laboratory, Vehicle Structure Technology Division

Reiko KOGANEI, Dr. Eng.

Researcher,

Vehicle Structure Technology Division

\section{Yasuhiro UMEHARA}

Researcher,

Running Gear Laboratory,

\section{Yusuke YAMANAGA}

Former Researcher, Running Gear Laboratory,

Railway vehicles are equipped with multiple oil dampers, whose characteristics have a great effect on riding comfort for passengers as well as on the safety of vehicles. The technical specifications of these dampers are therefore essential in vehicle design. We have developed test equipment to allow estimation of oil damper characteristics without the need to perform field tests, making it possible to operate an oil damper three-dimensionally to simulate the actual running conditions of railway vehicles. This paper gives an outline and describes the specifications of the test equipment and the automatic identification of oil damper characteristics.

Keywords: damper, test equipment, HILS, iteration, neural network

\section{Introduction}

Railway vehicles are equipped with multiple oil dampers, whose characteristics have a great effect on riding comfort for passengers as well as on the safety of vehicles. The technical specifications of these dampers are therefore essential in vehicle design.

JIS (Japanese Industrial Standards) E 4205 defines the measured force without the stiffness effect of the attachment as the damper force. However, dampers have elastic devices (such as rubber bushes) at both connecting points to enable them to tolerate three-dimensional vehicle motion, meaning that they generate three-dimensional forces that act not only in the principal axis direction but also in other directions. Furthermore, Shimomura [1] reports that the characteristics of the rubber bushes in anti-yaw dampers have a great effect on hunting stability. It is therefore very important to evaluate dampers under actual conditions.
Although field tests are an effective method of estimating vehicle characteristics, they can be very difficult to execute because of the large amounts of time and human resources involved. Furthermore, the lack of test lines in Japan means that field testing is usually executed on commercial lines, so extreme conditions are not permitted when executing tests. From this point of view, test equipment capable of simulating actual conditions is very effective in reducing the development period and improving the quality of development results. The RTRI is currently developing a virtual running-test environment using the HILS (Hardware In the Loop Simulation) technique. Damper test equipment is among the types used in this development project. It is possible to test any oil damper three-dimensionally to simulate the actual running conditions of railway vehicles. This paper gives an outline and describes the characteristics of the test equipment and automatic identification of oil damper characteristics.

Table 1 Damper specifications applicable to the damper test equipment

\begin{tabular}{|l|c|c|c|c|c|c|c|}
\hline & \multirow{2}{*}{$\begin{array}{c}\text { Max. } \\
\text { force }\end{array}$} & $\begin{array}{c}\text { Max. } \\
\text { speed }\end{array}$ & \multirow{2}{*}{$\begin{array}{c}\text { Max. test } \\
\text { frequency }(\mathrm{Hz})\end{array}$} & $\mathrm{X}$ & $\mathrm{Y}$ & $\mathrm{Z}$ & \multirow{2}{*}{ Direction } \\
\cline { 5 - 8 } & $(\mathrm{kN})$ & $(\mathrm{m} / \mathrm{s})$ & & & & & \\
\hline Lateral damper & 15 & 0.2 & 15 & 20 & 200 & 80 & $\mathrm{Y}$ \\
\hline Axle damper & 8 & 0.3 & 30 & 20 & 10 & 100 & $\mathrm{Z}$ \\
\hline $\begin{array}{l}\text { Anti-yaw damper } \\
\text { between cars }\end{array}$ & 22 & 0.2 & 10 & 200 & 200 & 80 & $\mathrm{X}$ \\
\hline Anti-yaw damper & 15 & 0.2 & 30 & 100 & 200 & 80 & $\mathrm{X}$ \\
\hline Anti-rolling damper & 10 & 0.1 & 10 & 20 & 200 & 160 & $\mathrm{Z}$ \\
\hline Anti-tilting damper & 5 & 0.3 & 2 & 20 & 300 & 20 & $\mathrm{Y}$ \\
\hline
\end{tabular}




\section{Outline of the damper test equipment}

The specifications of this damper equipment were set to allow installation of any damper applied to railway vehicles. Table 1 shows the damper specifications applicable to the equipment, which allows installation of test dampers in two directions, i.e. horizontally and vertically. There are three acting directions in actual dampers, longitudinal (X), lateral (Y) and vertical (Z). Accordingly, axis transform may be necessary in test execution. Figure 1 shows the damper equipment developed.

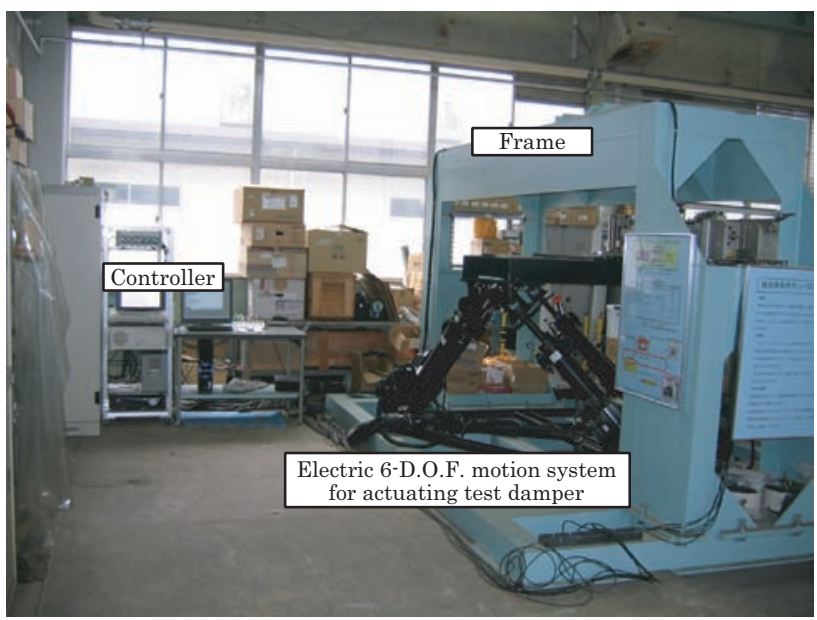

Fig. 1 External view of the test equipment

\subsection{Damper actuating mechanism}

We adopted a Stewart-platform-type electric 6-D.O.F. motion system (a kind of parallel-link mechanism) for the actuating damper (Fig. 2). This has the advantage of maintaining uniformity in all directions with respect to frequency performance and rigidity compared with a serial-link mechanism. Table 2 shows the specifications of the motion system.

During testing, the dampers are set between the motion system and the equipment frame. Figure 3 shows the installation of the test dampers.

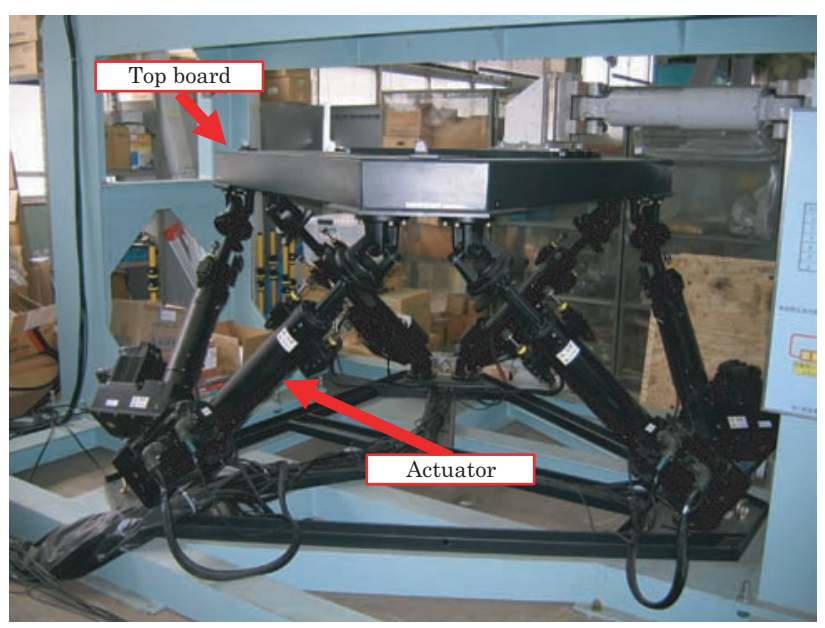

Fig. 2 Electric 6-D.O.F. motion system

Table 2 Specifications of the motion system

\begin{tabular}{|c|c|c|}
\hline & Motion system & Required specs. \\
\hline Motion limits & & \\
\hline Longitudinal & $\pm 200 \mathrm{~mm}$ & $\pm 100 \mathrm{~mm}$ \\
\hline Lateral & $\pm 195 \mathrm{~mm}$ & $\pm 150 \mathrm{~mm}$ \\
\hline Vertical & $\pm 190 \mathrm{~mm}$ & $80 \mathrm{~mm}$ \\
\hline Max. damping or thrust force & $22 \mathrm{kN}$ & $22 \mathrm{kN}$ \\
\hline Max. velocity & $0.45 \mathrm{~m} / \mathrm{s}$ & $0.3 \mathrm{~m} / \mathrm{s}$ \\
\hline
\end{tabular}

\subsection{Measurement device}

To measure three-dimensional forces and moment, we have chosen a six-component force transducer making use of a strain gage, which is installed between a damper bracket and the equipment frame. The rated capacity in the principal direction is up to $20 \mathrm{kN}$.

\subsection{Controller}

The equipment controller performs the two roles of motion order to the motion system and data acquisition from the six-component force transducer. To cooperate

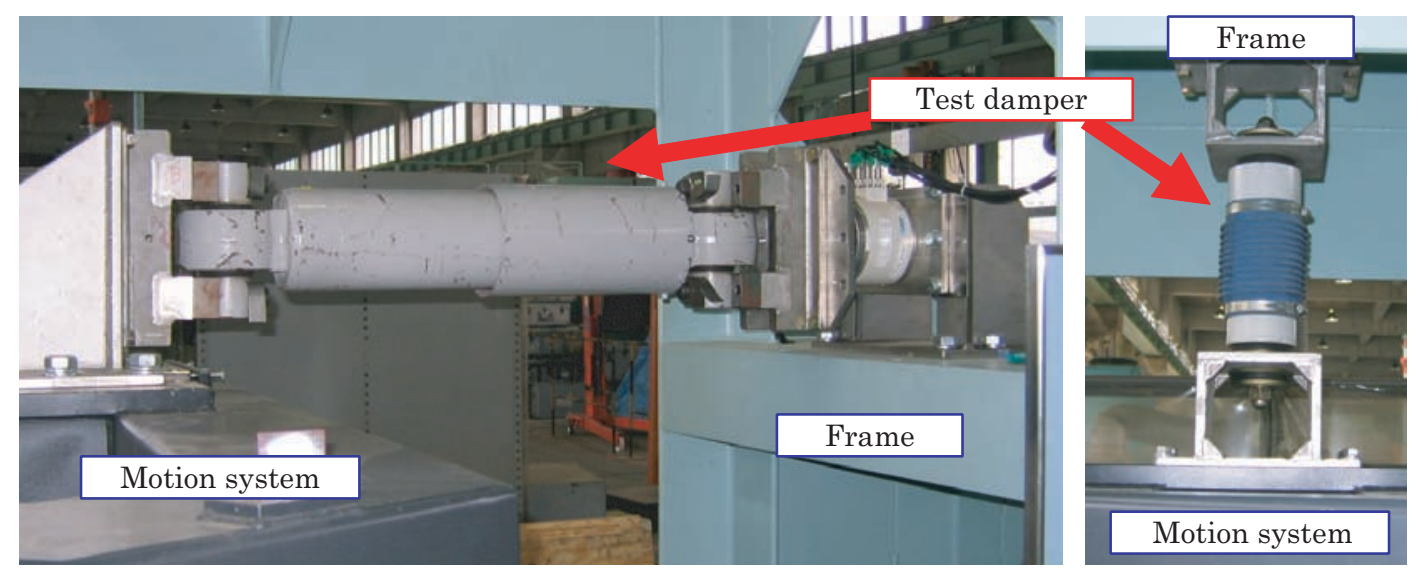

(a) Horizontal installation

Fig. 3 Installation of test dampers (b) Vertical installation 
with other computer simulators in HILS, it can be connected to an optical real-time network.

\section{Performance improvement of the test equipment}

\subsection{Introduction}

It is difficult for actual motion movement to be realized by the motion order without compensation in each time step. The motion system controls the top board by adjusting the actuator length, and some dynamical error exists between the motion order and the actual movement because of the motion transfer characteristics. In addition, static error may also exist because of the tolerance manufacturing and setting stage (Fig. 4).

Real-time simulation is executed in collaboration with the test equipment during HILS testing. It may be a critical issue from the standpoint of simulation accuracy and stability for the system to control the damper position properly in each time step without delay, otherwise the simulation may receive an erroneous response (in terms of forces) from the transducer.

The performance improvement test was executed as follows:

(1) Six actuators' length of the motion system was measured at the static movement order. The top-board movement without static error was then estimated from these lengths (referred to below as the estimated movement). If it agrees with the motion order, we can also evaluate the estimated movement dynamically in each time step.

(2) The top-board movement including static error was measured using theodolites at the static movement order (referred to below as the actual movement), and the relationship between the actual movement and the estimated movement (equal to the motion order from step (1)) was acquired. We can statically adjust the actual movement using this relationship.

(3) The characteristics of motion were acquired by measuring the actuators' length during dynamic operation, and the compensator was considered. The actual movement can also be adjusted dynamically together with step (2) using a compensator of appropriate design.

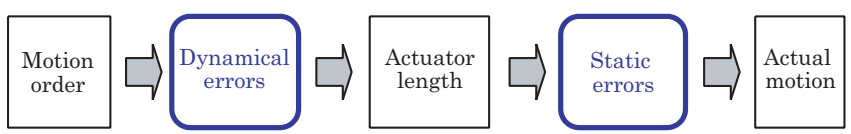

Fig. 4 Error factors between the motion order and the actual motion

\subsection{Estimation of top-board movement from the actuators' length}

The motion system controls the top-board movement by adjusting the length of each actuator that is set without tolerance as described above.

If we assume that the coordinates of the upper connecting point of the $i$-th actuator are given as $P_{U i}(t)=$
$\left(x_{U i}(t), y_{U i}(t), z_{U i}(t)\right)^{T}$, then the following equation can be obtained:

$$
P_{U i}(t)=R_{X}(\varphi(t)) R_{Y}(\theta(t)) R_{Z}(\phi(t)) P_{U i}(0)+(x(t), y(t), z(t))^{T}
$$

where $R_{X}, R_{Y}$ and $R_{Z}=$ rotation matrix with respect to fixed axes X, Y and Z; $x(t), y(t), z(t), \phi(t), \theta(t)$ and $\varphi(t)$ = estimated movement, translation and roll-pitch-yaw angle.

The squared length of the $i$-th actuator $L_{2 i}(t)$ is obtained by

$$
L_{2 i}(t)=\left(x_{U i}-x_{B i}\right)^{2}+\left(y_{U i}(t)-y_{B i}\right)^{2}+\left(z_{U i}(t)-z_{B i}\right)^{2}
$$

where $x_{B i}, y_{B i}$ and $z_{B i}=$ the coordinates at the fixed bottom connecting point of the $i$-th actuator. The estimated movement is obtained from the measured actuator length and equations (1) and (2) using numerical analysis.

The maximum errors of the estimated movement against the static motion order up to $\pm 150 \mathrm{~mm}$ (in the $\mathrm{X}$, $\mathrm{Y}$ and $\mathrm{Z}$ directions), \pm 9 degrees (roll, pitch and yaw angle) are $0.02 \mathrm{~mm}$ and 0.001 degrees, indicating that this method has sufficiently high accuracy for estimating the top-board movement at each time step.

\subsection{Measurement of actual top-board movement using theodolites}

Theoretically, the coordinates of the fixed measured points on the top board can be calculated by measuring the angles of direction and elevation of these points using two theodolites set at a known distance from each other. In reality though, the accuracy of such calculation results is not high because the measured angles introduce a certain level of error. The coordinates are therefore calculated using the following method:

(1) The coordinates at the foot of a perpendicular line from the measured point to the horizontal plane (including the first theodolite) are calculated using the angles of direction and the distance between the two theodlites. This foot point is referred to as $H$.

(2) The coordinates of the fixed measured point are calculated on the local coordinate system (whose origin is the first theodolite) from the angle of elevation of the first theodolite and the distance between the first theodolite and $H$.

(3) The coordinates of the second theodolite are calculated on the same local coordinate system as above from the angle of elevation of the second theodolite and the distance between the second theodolite and $H$.

Measurement errors appear as a dispersion of the second theodolite's coordinates, so we can check the dispersion of the results to confirm the validity of measurement.

Figure 5 shows actual movement error at static motion orders up to $\pm 150 \mathrm{~mm}$ (in the $\mathrm{X}, \mathrm{Y}$ and $\mathrm{Z}$ directions). These errors change linearly against the motion order. The actual movement can therefore be thought of as statically equal to the motion order with adjustment using these regression curves. 


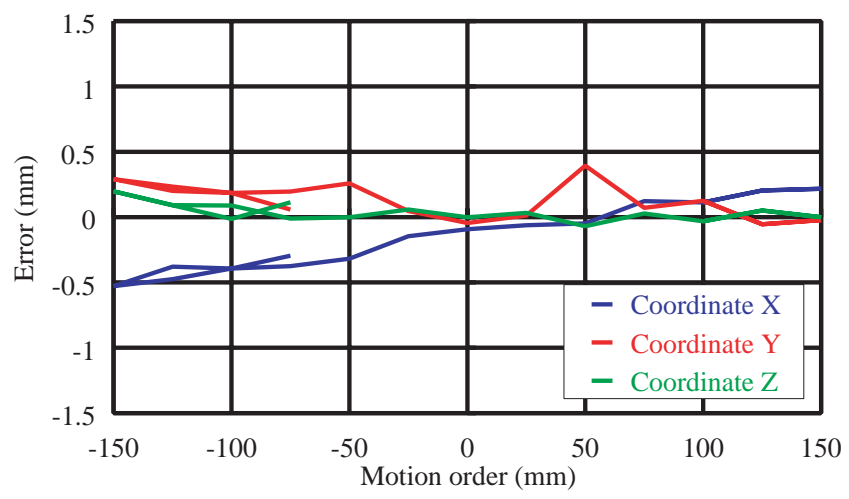

(a) Error distribution during translation along axis $\mathrm{X}$

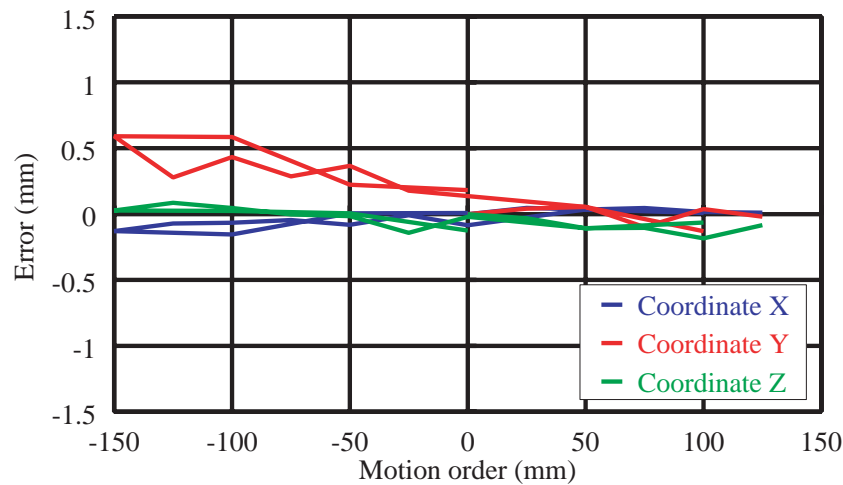

(b) Error distribution during translation along axis $\mathrm{Y}$

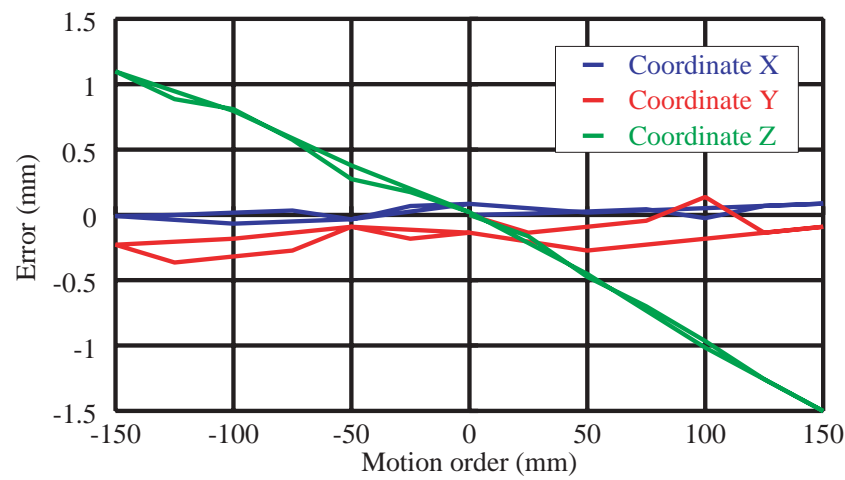

(c) Error distribution during translation along axis Z

Fig. 5 Actual movement error at static motion order

\subsection{Acquisition of motion characteristics and consideration of the compensator}

\subsubsection{Transfer function of the motion system}

The transfer function of the motion system is calculated from the ratio of the input-output cross spectrum to the input spectrum. Figure 6 shows the transfer function of the motion system calculated from random wave inputs along the $\mathrm{X}$-axis direction, where the half amplitude is up to $15 \mathrm{~mm}$ and the frequency is up to $3 \mathrm{~Hz}$.

\subsubsection{Consideration of the compensator}

There are two points of difficulty in designing a compensator applicable to HILS testing: the compensator

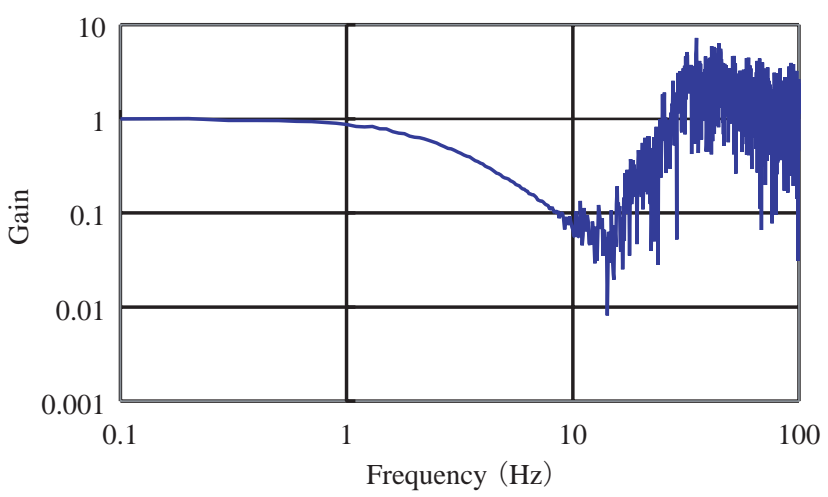

Fig. 6 Calculated transfer function for random wave inputs along the $\mathrm{X}$-axis direction

cannot use future inputs or preparative test results, because it receives an order at the very moment when the real-time simulator finishes calculating at each time step in HILS testing.

We designed the compensator to flatten the transfer characteristic of the motion system using the Yule-Walker technique, which is an IIR filter design method. The YuleWalker technique compensates only the gain characteristic, and does not always compensate the phase characteristic. However, the phase characteristic can generally be made flat by using another appropriate compensator with it. Basically, the compensator is represented by the inverse transfer function of the motion system. At this time, the calculated transfer function is filtered because

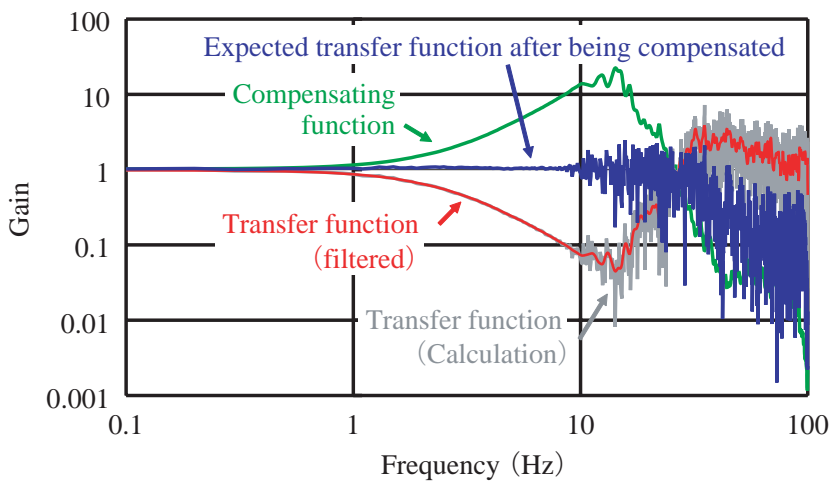

Fig. 7 Compensating function and expected transfer function

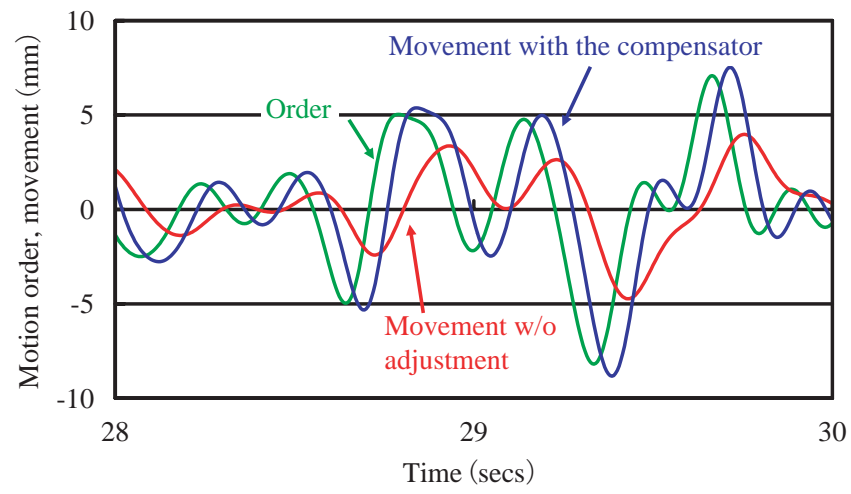

Fig. 8 Effect of the designed compensator for random wave inputs along the $\mathrm{X}$-axis direction 
of noise from the system, and gain characteristic beyond $30 \mathrm{~Hz}$ is intentionally cut off. Figure 7 shows the compensating function and the expected transfer function of the motion system after being compensated.

Figure 8 shows a time history of the motion results by compensated orders. Improvement of the phase characteristic over that of the gain characteristic is not seen, indicating time delays in the software that controls the motion system. Improvement of this software is therefore needed.

\section{Automatic identification of oil damper characteristics}

\subsection{Aim of automatic identification}

Although a virtual field-test environment enables actual devices to be combined with computer simulations for hybrid tests, it is not a realistic test method to install all devices in test equipment on board a vehicle. Most of these devices are therefore represented by computer simulation models.

Conventional modeling methodology does not provide highly accurate models in a wide range of simulation conditions for strongly non-linear devices such as dampers. This is because the methodology generates more characteristic-comprehensive models with fewer parameters using linearization and approximation in restricted coverage.

In automatic identification of damper characteristics, automatic generation of simulation models aims at a threestep development. The first is automatic generation of the actuating pattern for the damper test equipment from parameters such as stroke and designed damping force diagrams, which are shown in drawings. The second step involves data acquisition during the test with this pattern, and the final one consists of automatic generation of a simulation model. We have focused only on the relationship between input (damper displacement and velocity) and output (damper force) without paying attention to modeling a detailed damper interior structure.

\subsection{Neural network}

A neural network $[2,3]$ is a non-parametrical identification method, and is applied to automatic identification of damper specifications. Such networks constitute a parallel-distributed processing model developed by simulating simplified neural processing in the brain. The network is represented by connections between artificial neurons (Fig. 9), each of which has input weights and bias as its parameters. Its output to the adjacent neurons is calculated from the weighted sum of inputs with its own predefined function, and the final output on the output layer is the network output. A neural network automatically adjusts input weights and biases to minimize the error of the desired input-output pattern presented (known as the training sample). One of the most popular techniques for adjustment is back propagation, and this process is referred to as training.

Two types of neural network (the multi-layer feedforward type and the multi-layer recurrent type) are

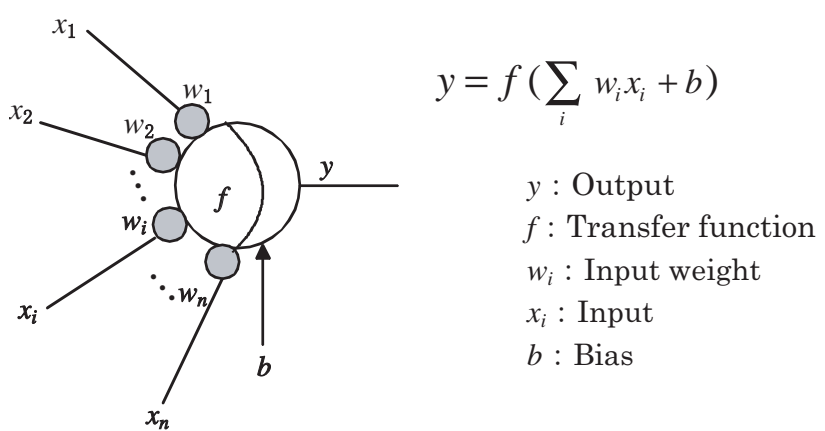

Fig. 9 Artificial neuron model

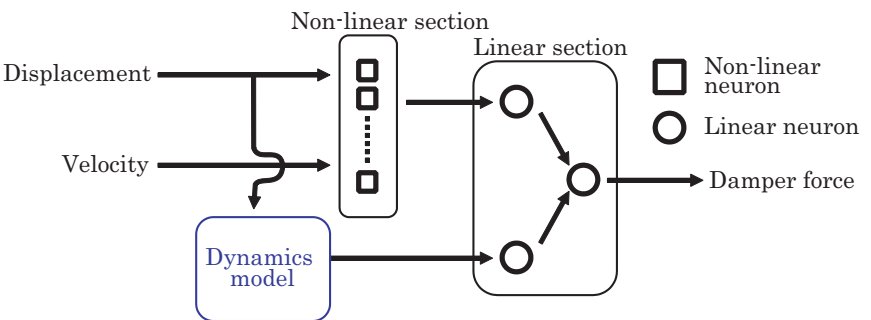

Fig. 10 Neural network with simplified dynamics model for identification

considered for application to identification. A recurrent neural network contains feedback loops, and enables training to be established with fewer neurons in timeseries-dependent systems. However, the feedback loops may cause undesirable output to continue once it occurs, and in the worst case diffused output may cause a fatal result. In contrast, a feedforward neural network needs many neurons for training in time-series-dependent systems, and avoids the propagation of undesirable output if it occurs.

We have established automatic identification with a multi-layer feedforward neural network using the damper displacement and speed as the network inputs and the force of the damper as the output. To improve the performance of the feedforward network, we have also used a simplified dynamics model that calculates the strain of the elastic devices installed at both ends of the damper as pre-work for preparing the network input (Fig. 10).

\subsection{Identification of the principal direction}

\subsubsection{Identification experiment procedure}

To confirm the validity of the identification method described above, we used a lateral damper installed on a Shinkansen bogie in an identification experiment of the principal direction. Two different types of sample were prepared (i.e. training and verifying samples), and the damper test equipment was used to certify that output from the trained neural network was equal to that of the verifying samples.

\subsubsection{Actuating data}

The trained neural network gives highly accurate output if the input data are within the training input range. However, if the data are outside this range, the 


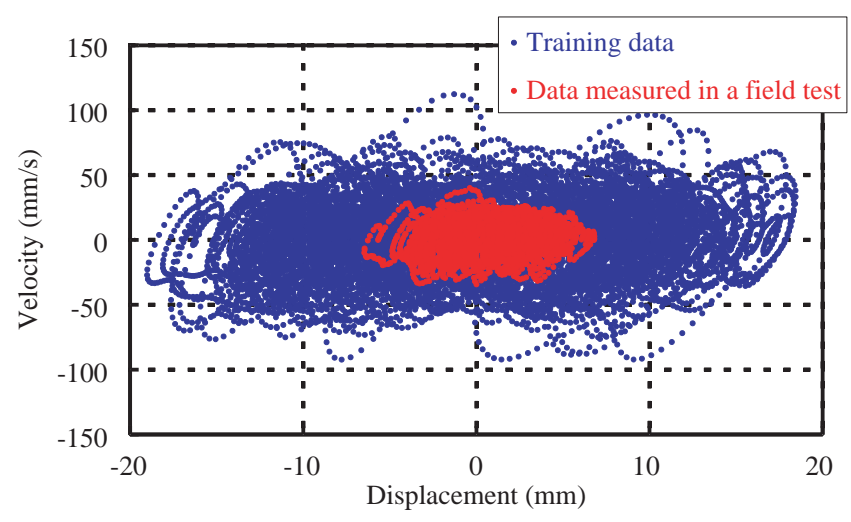

Fig. 11 Displacement and speed distribution in training data

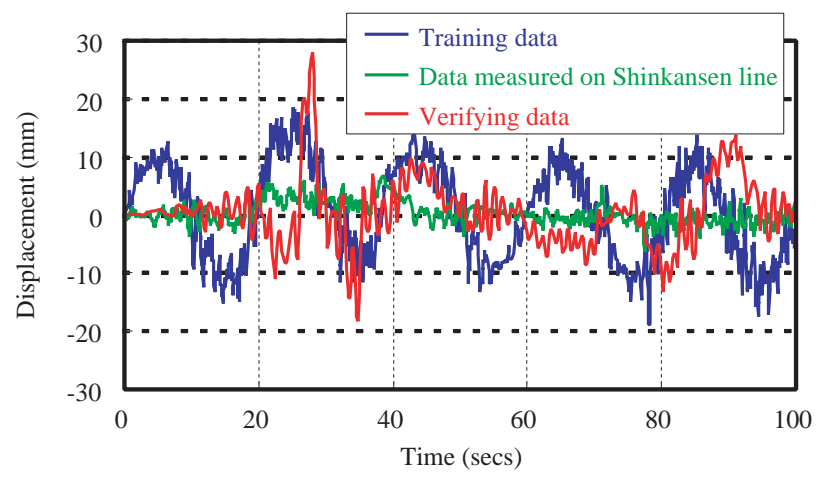

Fig. 12 Verifying data and training data

network often outputs very low-accuracy values because it recognizes these inputs as unknown patterns. It is therefore important that the range of training input data includes the data ranges used by the trained network. For this reason, actuating data to make training samples were prepared with a five-fold amplitude of the damper stroke obtained in a field test, and a sine wave (with a frequency of $0.05 \mathrm{~Hz}$ and a peak amplitude of $10 \mathrm{~mm}$ ) was applied to the data. The displacement and velocity distribution of the actuating data includes that of a field test on a Shinkansen line, as shown in Fig.11.

The actuating data for verification are non-correlative with those for training, and consist of measurements taken in field testing on a meter-gauged line. These are different from both the training data and the data measured in a field test on a Shinkansen line, as shown in Fig.12.

\subsubsection{Identification results}

We confirmed the extent to which the calculated outputs of the trained network (Fig. 10) will coincide with the measured force of the actual damper using test equipment and verifying data.

Figure 13 shows the results of the calculated outputs of the trained network. To confirm its effect, the calculated outputs of an ARX model (a popular parametrical identification method) are also shown in Fig. 13. These outputs are different from the measured data, especially

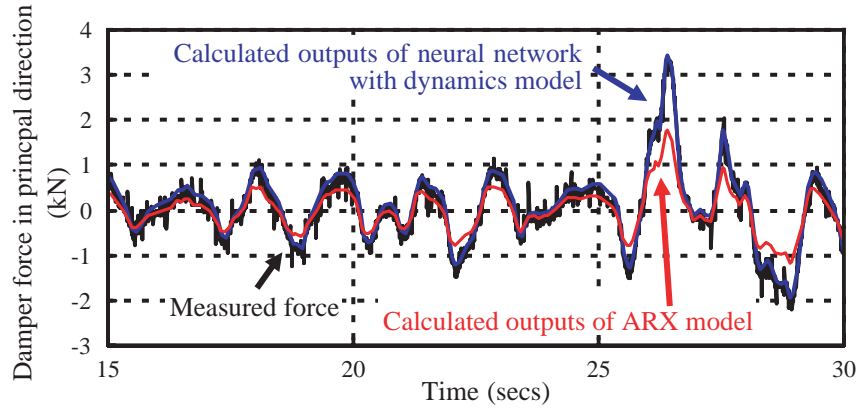

Fig. 13 Results of calculation by neural network

at the peaks. However, the calculated outputs of the neural network model are overall the same as the measured data, irrespective of the damper force amplitude.

\section{Conclusions}

(1) We have developed damper test equipment capable of simulating actual conditions. It can actuate any damper installed on vehicles three-dimensionally, and is capable of acting as a component of HILS to cooperate with real-time simulation.

(2) The relationship between the motion order and the actual movement has been clarified through performance improvement.

(3) We have acquired the characteristics of the motion system, and have designed a compensator to flatten the gain characteristic.

(4) We have executed an automatic characteristics identification experiment using a lateral damper. The proposed neural network model is effective.

We plan to improve the controller of this equipment to execute HILS testing, and to develop an automatic identification method for the characteristics of air suspension.

\section{Acknowledgment}

This work is financially supported in part by the Japanese Ministry of Land, Infrastructure and Transport.

\section{References}

[1] Shimomura, T., "Stability and riding comfort of highspeed vehicles," RRR, Vol. 61, No. 1, pp. 14-17, 2004 (in Japanese).

[2] Nagai, M. et al., "Identification and control of nonlinear active pneumatic suspension for railway vehicles using neural networks," Transactions of the Japan Society of Mechanical Engineers. C, Vol. 61, No. 586, pp. 2293-2298, 1995 (in Japanese).

[3] Kamiyama, K. et al., "Experimental Identification of Nonlinear Vibratory Systems by Neural Networks," Transactions of the Japan Society of Mechanical Engineers. $C$, Vol. 67, No. 663, pp. 3398-3404, 2001 (in Japanese). 